\title{
Scattering of GeV Electrons in the Framework of the Relativistic Hartree Approximation
}

\author{
A. Mariano, C. Barbero, \\ Departamento de Física, Facultad de Ciencias Exactas, \\ Universidad Nacional de la Plata, cc. 67, 1900 La Plata, Argentina \\ and F. Krmpotić \\ Instituto de Física, Universidade de São Paulo, C. P. 66318, 05315-970 São Paulo, SP, Brazil
}

Received on 10 March, 2004

\begin{abstract}
The high momentum transfer electron-nucleus scattering cross section is evaluated within the plane wave impulse approximation (PWIA) supplemented by the relativistic Hartree approximation (RHA). Binding effects on the struck nucleon are introduced through the scalar and vector meson-exchange fields within the framework of quantum hadrodynamics. This model naturally satisfies the current conservation, with the off-shell nucleons behaving as being on the mass shell but having an effective mass. The nucleon inelastic response is included via different parameterizations of the structure function measured at SLAC, while the smearing of the Fermi surface is introduced through a momentum distribution obtained from a perturbative nuclear matter calculation. Recent CEBAF data on inclusive scattering of $4.05 \mathrm{GeV}$ electrons on ${ }^{56} \mathrm{Fe}$ are well reproduced for all measured geometries by the first time. Scaling effects are analyzed as well, and the scaling properties of the nuclear response in terms of the $y$ variable associated to the PWIA within the RHA framework are discussed. The theoretical scaling function obtained in our approach also significantly improves previous PWIA calculations within the multi-GeV electron scattering regime, and describes properly the CEBAF scaling data.
\end{abstract}

The inclusive electron-nucleus scattering is a powerful tool for investigating the effective constituents of hadronic matter and their dynamics. Experiments performed in different regions of the square four-momentum $q^{2}(q \equiv(\omega, \mathbf{q}))$ and energy $\omega$ transfers, provide information on the nuclei constituents and diverse excitation mechanisms. They are: (i) the quasielastic scattering region, $\omega \leq Q^{2} / 2 M$, with $M$ being the nucleon mass and $Q^{2} \equiv-q^{2}>0$, where experimental data can be analyzed in terms of scaling variables providing information on nuclear dynamics and the nucleon momentum distribution; (ii) the inelastic scattering region, $\omega \geq Q^{2} / 2 M+M_{\pi}$, with $M_{\pi}$ being the pion mass, where nucleon resonances are excited and medium induced modifications of their properties can be studied; (iii) the deep inelastic scattering region, $W \equiv \sqrt{(p+q)^{2}} \geq 2$ $\mathrm{GeV}, Q^{2} \geq 1(\mathrm{GeV} / \mathrm{c})^{2}$, being $p$ the initial nucleon fourmomentum, where possible modifications of quarks and gluon distributions in the nucleon induced by the medium can be investigated. In the later case the response function also reflects the presence of 6-quark bags in the nuclear wave function. reads

In the Born approximation the differential cross section

$$
\frac{d^{2} \sigma}{d \Omega^{\prime} d \epsilon^{\prime}}=\frac{\alpha^{2}}{\mathrm{q}^{4}} \frac{\mathrm{k}^{\prime}}{\mathrm{k}} L^{\mu \nu} W_{\mu \nu},
$$

where $L^{\mu \nu}\left(k, k^{\prime}\right)$ is the lepton tensor describing incoming and outgoing plane-wave electron states with four- momentum $k=\left(\epsilon \equiv \sqrt{\mathbf{k}^{2}+m^{2}}, \mathbf{k}\right)$ and $k^{\prime}=\left(\epsilon^{\prime} \equiv\right.$ $\left.\sqrt{\mathbf{k}^{\prime 2}+m^{2}}, \mathbf{k}^{\prime}\right)$, respectively, where $\mathrm{k} \equiv|\mathbf{k}|, \mathrm{k}^{\prime} \equiv\left|\mathbf{k}^{\prime}\right|$, and $\alpha=\frac{e^{2}}{4 \pi}$ is the fine structure constant and $\Omega^{\prime} \equiv(\theta, \phi)$ the scattering angle. The most general form for $W_{\mu \nu}$ satisfying simultaneously Lorentz invariance, gauge invariance $\left(q^{\mu} W_{\mu \nu}=0\right)$ and the parity conservation is

$$
\begin{aligned}
& W_{\mu \nu}(p, q)=W_{1}\left(q^{2}, p \cdot q\right)\left[-g_{\mu \nu}+\frac{q_{\mu} q_{\nu}}{q^{2}}\right] \\
& +W_{2}\left(q^{2}, p \cdot q\right)\left[\frac{p_{\mu}}{M}-\frac{p \cdot q}{M} \frac{q_{\mu}}{q^{2}}\right]\left[\frac{p_{\nu}}{M}-\frac{p \cdot q}{M} \frac{q_{\nu}}{q^{2}}\right],
\end{aligned}
$$

where $W_{1,2}$ are Lorentz scalars. For a nuclear target at rest $\left(M \equiv M_{A}\right)$ we have $p \equiv\left(M_{A}, 0\right)$ and $p \cdot q \equiv M_{A} \omega$, which when combined with the above two equations lead to

$$
\begin{aligned}
\frac{d^{2} \sigma}{d \Omega^{\prime} d \epsilon^{\prime}} & =\frac{d \sigma_{M}}{d \Omega^{\prime}}\left[\frac{Q^{2}}{\mathrm{q}^{2}} W_{L}^{A}(\omega, \mathbf{q})\right. \\
& \left.+\left(\frac{1}{2} \frac{Q^{2}}{\mathrm{q}^{2}}+\tan ^{2} \frac{\theta}{2}\right) W_{T}^{A}(\omega, \mathbf{q})\right],
\end{aligned}
$$

where $A \equiv(N, Z), d \sigma_{M} / d \Omega^{\prime}$ is the Mott cross section, and where we have used the well known longitudinal $\left(W_{L}^{A}\right)$ and transverse $\left(W_{T}^{A}\right)$ response functions

$$
\begin{aligned}
& W_{L}^{A}(\omega, \mathbf{q})=\frac{Q^{2}}{\mathrm{q}^{2}} W_{2}^{A}(\omega, \mathbf{q})-W_{1}^{A}(\omega, \mathbf{q}), \\
& W_{T}^{A}(\omega, \mathbf{q})=2 W_{1}^{A}(\omega, \mathbf{q}),
\end{aligned}
$$


which must be evaluated over the entire range $0 \leq \omega \leq \mathrm{q}$.

The PWIA [1], is inspired on the fact that the electron probes only a small region of dimensions $1 / \mathrm{q}$ and is based on the following assumptions:

i) the nuclear current operator can be written as the sum of one-body nucleon currents;

ii) the target decays virtually into an on-shell (A-1) nucleus (spectator) and an off-shell $\left(p^{2} \neq M^{2}\right)$ struck nucleon; and

iii) the nucleon that absorbs the photon is the same that leaves the target without interaction with the spectator.

To deal with the off-shell effects, we adopt in this work an approximation that keeps simultaneously the gauge invariance and the covariant kinematics of the struck nucleon. The nucleon will be bound trough the interaction with the scalar $\phi$ and vector $V_{\mu}$ mesons fields, within the framework of quantum hadrodynamics (QHDI)[2]. The nucleon single particle spectrum reads

$$
p_{0}=\Sigma_{0}^{V}+E_{\mathbf{p}}^{*}
$$

with $E_{\mathbf{p}}^{*}=\sqrt{\mathbf{p}^{2}+M^{* 2}}$, being

$$
M^{*} \equiv M+\Sigma_{R H A}^{S}\left(C_{S}, M^{*}\right) .
$$

$M^{*}<M$ is the effective mass acquired by the nucleon by the action of the attractive scalar field and is determined self- consistently through the scalar self-energy $\Sigma_{R H A}^{S}$, within the Hartree approximation. The vector self-energy $\Sigma_{0}^{V} \equiv$ $C_{V}^{2} \rho_{B} / M^{2}$ accounts for the action of the repulsive vector field, and the two free parameters $C_{S}$ and $C_{V}$ depend on the meson coupling constants and masses being fixed to reproduce the experimental binding energy per nucleon at the baryon density $\rho_{B}$ for the normal nuclear matter [3].

In the PWIA + RHA approximation, the response tensor can be expressed in the laboratory system as

$$
W_{\mu \nu}^{A}(q)=2 \sum_{m_{t}} \int d \mathbf{p} \frac{M^{*}}{E_{\mathbf{p}}^{*}} n^{m_{t}}(\mathbf{p}) w_{\mu \nu}^{m_{t}}\left(p^{*}, q\right),
$$

being

$$
\begin{aligned}
& w_{\mu \nu}^{m_{t}}\left(p^{*}, q\right)=w_{1}^{m_{t}}\left(Q^{2}, \nu^{*}\right)\left[-g_{\mu \nu}+\frac{q_{\mu} q_{\nu}}{q^{2}}\right] \\
& +w_{2}^{m_{t}}\left(Q^{2}, \nu^{*}\right)\left[\frac{p_{\mu}^{*}}{M^{*}}-\nu^{*} \frac{q_{\mu}}{q^{2}}\right]\left[\frac{p_{\nu}^{*}}{M^{*}}-\nu^{*} \frac{q_{\nu}}{q^{2}}\right],
\end{aligned}
$$

where $m_{t} \equiv p(n)$ for protons (neutrons). This equation has the same form as the previously defined for an on-shell nucleon but with an effective mass $M^{*}$, which indicates that nuclear electromagnetic current is still conserved and the gauge invariance preserved [4]. Also we have

$$
\begin{aligned}
W_{L}^{A}(\omega, \mathbf{q}) & =2 \sum_{m_{t}} \int d \mathbf{p} n^{m_{t}}(\mathbf{p}) \frac{M^{*}}{E_{\mathbf{p}}^{*}}\left[\frac{\mathrm{q}^{2}}{Q^{2}}\left(1+\frac{p_{z}}{M^{*}} \frac{Q^{2}}{\mathrm{q} \nu^{*}}\right)^{2}\right. \\
& \left.\times\left(\frac{\nu^{*}}{\omega}\right)^{2} w_{2}^{m_{t}}\left(Q^{2}, \nu^{*}\right)-w_{1}^{m_{t}}\left(Q^{2}, \nu^{*}\right)\right], \\
W_{T}^{A}(\omega, \mathbf{q}) & =2 \sum_{m_{t}} \int d \mathbf{p} n^{m_{t}}(\mathbf{p}) \frac{M^{*}}{E_{\mathbf{p}}^{*}}\left[w_{1}^{m_{t}}\left(Q^{2}, \nu^{*}\right)\right. \\
& \left.+\left(\frac{p_{x}}{M^{*}}\right)^{2} w_{2}^{m_{t}}\left(Q^{2}, \nu^{*}\right)\right],
\end{aligned}
$$

where $q \equiv|\mathbf{q}|$. This result exhibits our prescription: $w_{1,2}^{\text {off-shell }}\left(Q^{2}, \nu\right)=w_{1,2}^{\text {on-shell }}\left(Q^{2}, \nu^{*}\right)$, where $\nu \equiv p$. $q / M \rightarrow \nu^{*} \equiv p \cdot q / M^{*}$, as a consequence of using the RHA to describe the struck nucleon. As said before, the probability for exciting the nucleon becomes important for $Q^{2}>1(\mathrm{GeV} / \mathrm{c})^{2}$. Thus, we cast $w_{1,2}^{m_{t}}$ in the form

$$
w_{1,2}^{m_{t}}=w_{e 1,2}^{m_{t}}+w_{i 1,2}^{m_{t}},
$$

where $w_{e 1,2}^{m_{t}}$ and $w_{i 1,2}^{m_{t}}$ are the elastic and inelastic nucleon responses, which will be both affected for the on-shell to off-shell mentioned prescription. For the functions $w_{i 1,2}^{m_{t}}$ we assume two different parametric fits obtained from the SLAC data on $p\left(e, e^{\prime}\right) p^{\prime}$ and $d\left(e, e^{\prime}\right) d^{\prime}$ reactions. One of the parameterizations was found by Bodek et al. [5] in the kinematical range $1<Q^{2}<20(\mathrm{GeV} / \mathrm{c})^{2}$ and $0.1 \leq$ $x \equiv Q^{2} /(2 M \omega) \leq 0.77$. The other one was reported by Whitlow [6], and corresponds to the range $0.6<Q^{2}<30$ $(\mathrm{GeV} / \mathrm{c})^{2}$ and $0.06 \leq x \leq 0.9$. Finally, the inclusive cross section reads

$$
\frac{d^{2} \sigma}{d \Omega^{\prime} d \epsilon^{\prime}}=\frac{d^{2} \sigma_{e}}{d \Omega^{\prime} d \epsilon^{\prime}}+\frac{d^{2} \sigma_{i}}{d \Omega^{\prime} d \epsilon^{\prime}}
$$

where the contributions coming from the elastic and inelastic nucleon responses are written separately.

The nucleon momentum distribution $n^{m_{t}}(\mathbf{p})$ has been evaluated as follows. First, the surface effects are supposed to be of minor importance and we adopt a non-relativistic 


$$
\begin{aligned}
\left|0_{A}\right\rangle & =\mathcal{N}\left[|0 p 0 h\rangle-\frac{1}{(2 !)^{2}} \sum_{p^{\prime} s, h^{\prime} s} \frac{\langle 2 p 2 h|\hat{V}| 0 p 0 h\rangle}{E_{2 p 2 h}}|2 p 2 h\rangle\right. \\
& \left.+\frac{1}{(4 !)^{2}} \sum_{p^{\prime} s, h^{\prime} s} \frac{\langle 4 p 4 h|\hat{V}| 2 p 2 h\rangle\langle 2 p 2 h|\hat{V}| 0 p 0 h\rangle}{E_{2 p 2 h} E_{4 p 4 h}}|4 p 4 h\rangle\right]
\end{aligned}
$$

where $\hat{V}$ is the residual interaction. This "minimum" perturbative scheme allows to include the norm correction $\mathcal{N}=$ $\left\langle 0_{A} \mid 0_{A}\right\rangle^{-1}$, avoiding in this way contributions from unbalanced disconnected diagrams. We get

$$
n^{m_{t}}(\mathbf{p})=\alpha^{m_{t}} n(\mathrm{p}) ; n(\mathrm{p})=\theta(1-\mathrm{p})+\delta n(\mathrm{p}),
$$

where $\mathrm{p} \equiv|\mathbf{p}|$ is measured in units of the Fermi momentum $\mathrm{p}_{F}$ and $\alpha^{m_{t}}=3 N^{m_{t}} / 4 \pi \mathrm{p}_{F}^{3} \cdot \theta(1-\mathrm{p})$ is the usual $0 \mathrm{p} 0 \mathrm{~h}$ Fermi step function, while

$$
\delta n(\mathrm{p})=\delta n^{(2)}(\mathrm{p})+\delta n^{(4 C)}(\mathrm{p}),
$$

contains the $2 \mathrm{p} 2 \mathrm{~h}$ and $4 \mathrm{p} 4 \mathrm{~h}$ contributions that deplete the Fermi surface, as illustrated in Fig. 1. The superscript $C$ indicates that only the "connected" $4 \mathrm{p} 4 \mathrm{~h}$ diagrams have to be included. For the residual interaction we use

$$
\hat{V}(\mathrm{q})=\sum_{I} V^{I}(\mathrm{q}) \mathbf{O}_{1}^{I}(\hat{\mathbf{q}}) \cdot \mathbf{O}_{2}^{I}(-\hat{\mathbf{q}})
$$

where the quantum numbers $I=T, S, J$ stand, respectively, for the isospin, the spin and the total angular momentum. The operators $\mathbf{O}^{I}(\hat{\mathbf{q}})$ are defined as

$$
\begin{aligned}
& \mathbf{O}^{000}(\hat{\mathbf{q}})=1 ; \quad \mathbf{O}^{010}(\hat{\mathbf{q}})=i(\hat{\mathbf{q}} \cdot \boldsymbol{\sigma}) ; \quad \mathbf{O}^{011}(\hat{\mathbf{q}})=(\hat{\mathbf{q}} \times \boldsymbol{\sigma}) \\
& \mathbf{O}^{100}(\hat{\mathbf{q}})=\boldsymbol{\tau} ; \quad \mathbf{O}^{110}(\hat{\mathbf{q}})=i(\hat{\mathbf{q}} \cdot \boldsymbol{\sigma}) \boldsymbol{\tau} ; \quad \mathbf{O}^{111}(\hat{\mathbf{q}})=(\hat{\mathbf{q}} \times \boldsymbol{\sigma}) \boldsymbol{\tau}
\end{aligned}
$$

and a Landau-Migdal parameterization plus a the static one pion exchange potential is adopted for the strengths $V^{I}$ (q) [7].

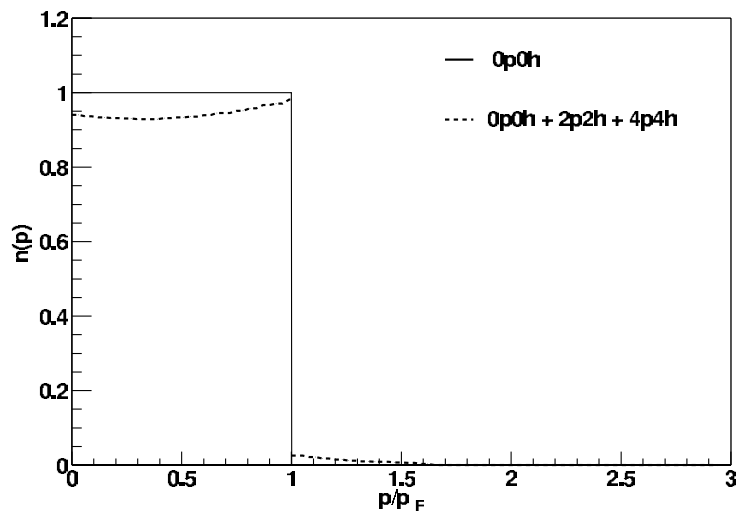

Figure 1. Momentum distribution function $n(p)=\theta\left(\mathrm{p}_{F}-\mathrm{p}\right)+$ $\delta n(\mathrm{p})$. The unperturbed step function corresponds to a pure $0 \mathrm{p} 0 \mathrm{~h}$ uncorrelated ground state and is indicated by full lines. The depletion of $n(\mathrm{p})$ comes form the admixtures of $2 \mathrm{p} 2 \mathrm{~h}$ and $4 \mathrm{p} 4 \mathrm{~h}$ excitations and is represented by dashed lines.
The scaling function is defined as

$$
\begin{aligned}
& F(\omega, \mathbf{q})=\frac{d^{2} \sigma(\omega, \mathbf{q})}{d \Omega^{\prime} d \epsilon^{\prime}} \frac{\mathbf{q}}{E_{\mathbf{p}_{\text {min }}}+\omega} \\
& \left(N \frac{d \sigma_{e}^{p}\left(\mathbf{p}_{\text {min }}, \omega, \mathbf{q}\right)}{d \Omega^{\prime}}+Z \frac{d \sigma_{e}^{n}\left(\mathrm{p}_{\text {min }}, \omega, \mathbf{q}\right)}{d \Omega^{\prime}}\right)^{-1},
\end{aligned}
$$

where $p_{\min }=|y|$, being $y$ the scaling variable

$$
y=-\frac{\mathrm{q}}{2}+\frac{\omega}{2} \sqrt{\frac{4 M^{* 2}}{Q^{2}}+1}
$$

obtained from the energy-conservation relation

$$
\omega=\sqrt{(\mathrm{q}+y)^{2}+M^{* 2}}-\sqrt{y^{2}+M^{* 2}}
$$

for a fixed $(\omega, \mathbf{q})$ pair. 
At high momentum transfers $\left(Q^{2}>1(\mathrm{GeV} / \mathrm{c})^{2}\right)$ and $y<0$, we get

$$
F(y)=2 \pi \int_{|y|}^{\infty} d \mathrm{pp} \sum_{m_{t}} n^{m_{t}}(\mathbf{p})
$$

which indicates that, for $Q^{2} \rightarrow \infty, F$ scales in $y$, i.e., it depends only on $y$ and not on $(\omega, q)$ or $(\omega, \theta)$ separately. Thus it should be approximately constant for a fixed value of $y$. In Figs. 2, 3 and 4 are confronted our theoretical results for the differential cross section in ${ }^{56} \mathrm{Fe}$ with the CEBAF experimental data [8] when the scattered electron are detected at angles $\theta$ of $15^{\circ}, 23^{\circ}, 30^{\circ}, 37^{\circ}, 45^{\circ}, 55^{\circ}$ and $74^{\circ}$. We evaluate as well the scaling function $F(\omega, \mathbf{q})$ as a function of the scaling variable $y$, that naturally appears in the model.

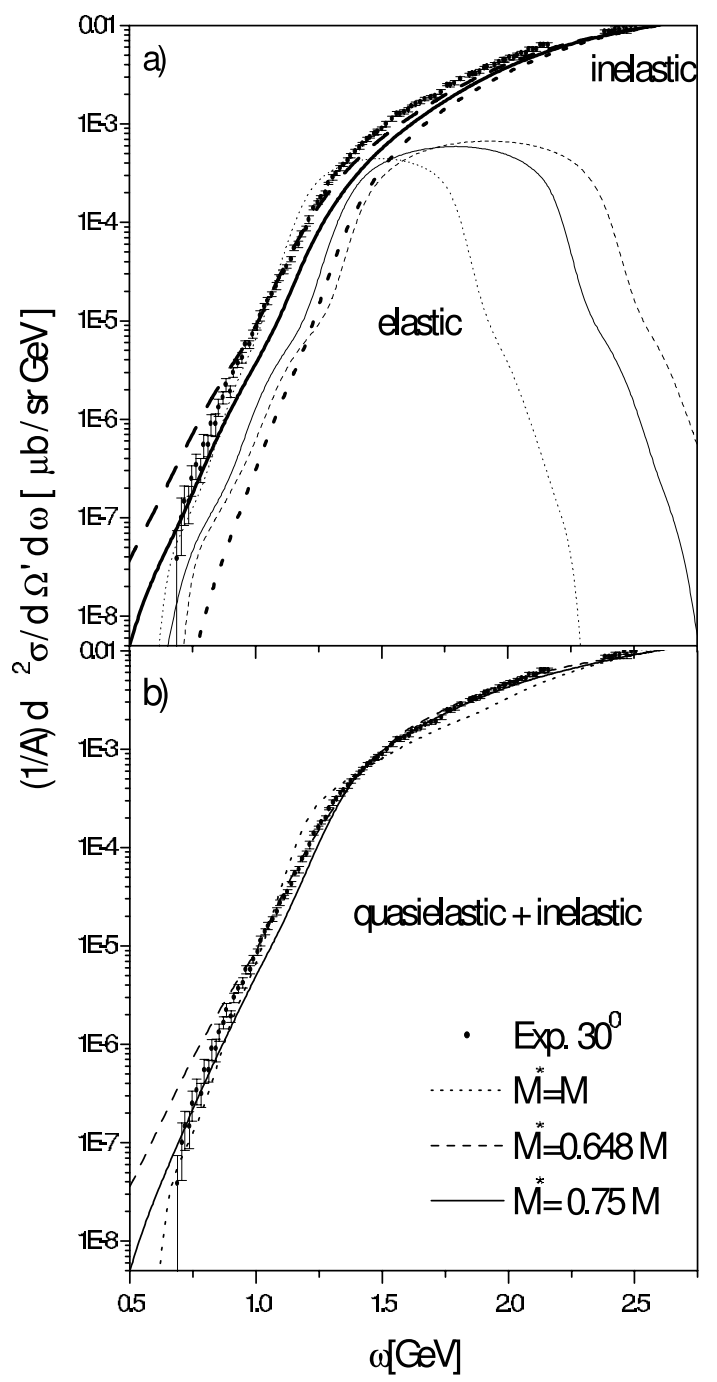

Figure 2. Sensibility of the per nucleon cross section in ${ }^{56} \mathrm{Fe}$ on the effective mass $M^{*}$. In the panel a) are shown separately the elastic (thin lines) and inelastic (thick lines) cross sections. In the panel b) are displayed the corresponding total cross sections. The data are from CEBAF.

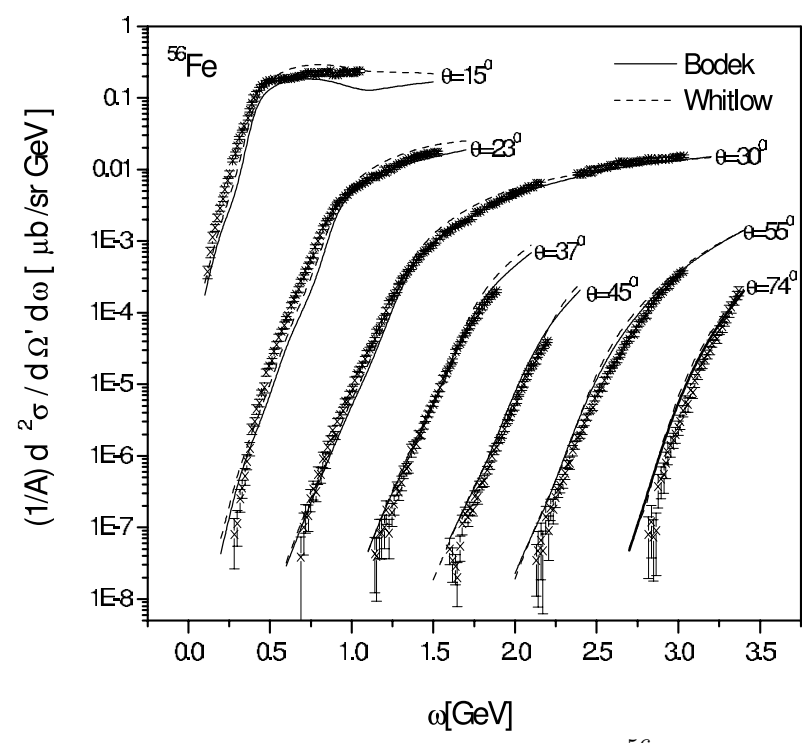

Figure 3. Calculated and measured cross sections in ${ }^{56} \mathrm{Fe}$ for different angles $\theta$. Theoretical results for both Bodek's (thin lines) and Withlow (thick lines) fittings of the inelastic nucleon response are exhibited. For the effective mass was used the value $M^{*}=$ 0.74 which is obtained in the relativistic Hartree approximation.

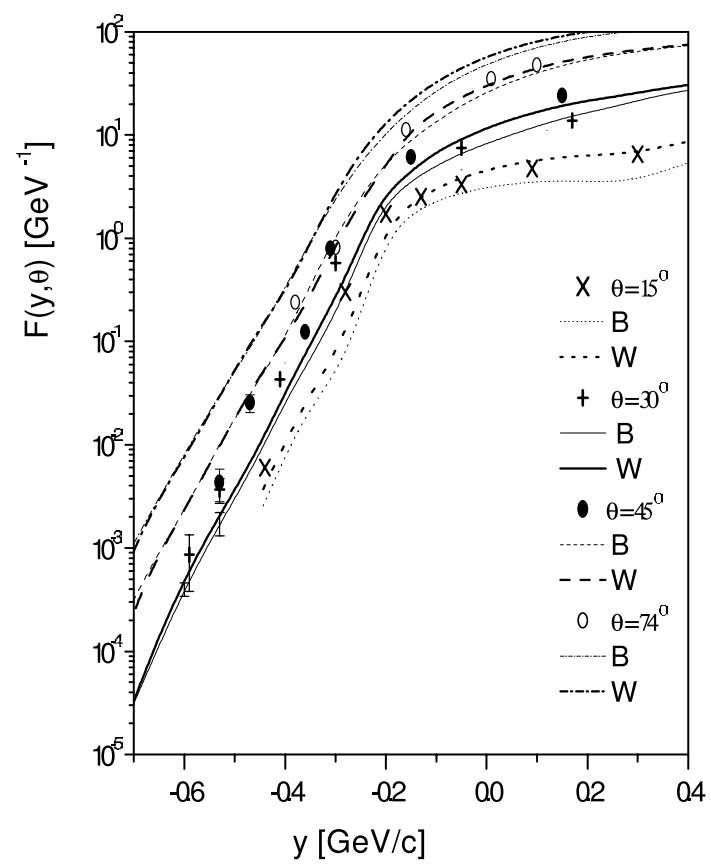

Figure 4. The scaling function $F(y, \theta)$ for ${ }^{56} \mathrm{Fe}$ a as function of the scaling variable $y$ for scattering angles $\theta=15^{\circ}, 30^{\circ}, 45^{\circ}$ and $74^{\circ}$. The experimental data are from CEBAF and are indicated by points, while the results corresponding to the fits of Bodek (B) and Whitlow (W) are given by thin and thick lines, respectively. A rearrangement of data is necessary since the scaling variable and the nucleon elastic cross sections in our model are different from those adopted in the CEBAF experiment.

In summary, to treat the scattering of GeV electrons by nuclei we have implemented a new version of the PWIA approach. The FSI has also be included to some extent. This is achieved by taking into account the binding effects of the struck nucleon in both the initial and final states. The relativistic kinematics is treated within the relativistic Hartree 
approximation that leads to better results than the plain relativistic mean field approach. In this picture, the binding effects are included through the effective nucleon mass $M^{*}=0.74 M$, and current conservation is naturally preserved without ad-hoc modifications in the structure functions. More precisely, in order to treat the scattering of $\mathrm{GeV}$ electrons we have pursued in the PWIA approach by introducing in a very simple way the relativistic effects the FSI and a new momentum distribution for the nucleons. The model does not pretend to substitute more evolved theoretical treatments; it merely yields a consistent and simple implementation of the PWIA which is able to reproduce satisfactory the full set of presently available data.

\section{References}

[1] C. Ciofi degli Atti and S. Simula, Phys. Rev. C43, 1155 (1991). C. Ciofi degli Atti, D.B. Day and S. Liuti, Phys. Rev. C46, 1045 (1991).

[2] B. D. Serot and J. D. Walecka, Adv. Nucl. Phys. 16, 1 (1986).

[3] J. D. Walecka, Theoretical Nuclear and Subnuclear Physics, ch. 39, Oxford University Press, New York, 1995.

[4] T. C. Ferrée and D. S. Koltun, Phys. Rev. C55, 253 (1997).

[5] A. Bodek and J. L. Ritchie, Phys. Rev. D 23, 1070 (1981).

[6] L. W. Whitlow, Ph.D. thesis, SLAC Report No. 357, 1990.

[7] A. Mariano, F. Krmpotic, and AFR. de Toledo Piza, Phys. Rev. C53, 1664 (1996).

[8] J. R. Arrington et al., Phys. Rev. Lett. 82, 2056 (1999). 